FIMAT-1/96

\title{
Can fractal-like spectra be experimentally observed in aperiodic superlattices?
}

\author{
Enrique Maciá and Francisco Domínguez-Adame \\ Departamento de Física de Materiales, Facultad de Físicas, Universidad Complutense, \\ E-28040 Madrid, Spain
}

\begin{abstract}
We numerically investigate the effects of inhomogeneities in the energy spectrum of aperiodic semiconductor superlattices, focusing our attention on Thue-Morse and Fibonacci sequences. In the absence of disorder, the corresponding electronic spectra are self-similar. The presence of certain degree of randomness, due to imperfections occurring during the growth processes, gives rise to a progressive loss of quantum coherence, smearing out the finer details of the energy spectra predicted for perfect aperiodic superlattices and spurring the onset of electron localization. However, depending on the degree of disorder introduced, a critical size for the system exists, below which peculiar transport properties, related to the pre-fractal nature of the energy spectrum, may be measured.
\end{abstract}

PACS numbers: 73.20.Dx, 71.50.+t, 73.61.Ey, 85.42.+m 


\section{INTRODUCTION}

One of the most appealing motivations for the experimental study of aperiodic superlattices (ASLs) arranged according to Fibonacci [1] and Thue-Morse [2] sequences is the theoretical prediction that these systems exhibit a highly-fragmented energy spectrum displaying self-similar patterns [3-5]. From a strict mathematical perspective, it has been proven that the spectra of both Fibonacci and Thue-Morse lattices are Cantor sets in the thermodynamic limit [6, [7]. From an experimental point of view, however, two major limitations appear to validate their peculiar fractal nature.

In the first place, it is not possible to fabricate perfect aperiodic structures. Although X-ray diffraction studies show that the characteristic structural order of ASLs is preserved under moderately large growth fluctuations [8]. the way this robust aperiodic order can influence the transport properties of actual, defective ASLs has not been received a proper treatment in the literature yet. In this sense, the observation of inhibition of vertical transport in periodic superlattices with intentional disorder [9], in agreement with the theory of localization in one-dimensional disordered systems, suggests the possible existence of a competition between the long-range aperiodic order and the unintentional short-range disorder and opens new perspectives [10].

In the second place, even in the most favorable experimental conditions, only finite arrangements with a limited number of layers can be manufactured. In this sense, the observation of fragmentation patterns in the energy spectra of short ASLs using different experimental techniques [11], encourages further analyses as to whether fractal-like spectra are to be expected in actual ASLs with an increasingly large number of layers.

Two important questions then follow quite naturally: First, what are the effects of unintentional disorder in the splitting scheme of the energy spectrum of ASLs? Second, what are the finite size effects on their fractal-like properties? In this paper we address these questions by means of the study of the phase diagram, the localization length and the bandwidth-scaling of the energy spectra. Our results indicate that small fluctuations in 
the sequential deposition of layers considerably smear out the self-similarity of the energy spectra on increasing the system size. Thus, we conclude that fractal-like spectra with a richness of finer details such those found by numerical analyses of perfect ASLs are not to be expected in large ASLs, but quantum coherence is strong enough in short systems to give rise to a measurable hierarchical set of subminibands in the electronic spectra and to influence its related transport properties.

\section{MODEL}

We consider quantum-well based GaAs- $\mathrm{Ga}_{1-x} \mathrm{Al}_{x} \mathrm{As}$ superlattices with the same barrier thickness $b$ in the whole sample. The height of the barrier for electrons is given by the conduction-band offset at the interfaces. We take the origin of electron energies at the GaAs conduction-band edge. The thickness of each quantum-well is $\Delta z_{n}-b \equiv z_{n}-z_{n-1}-b, z_{n}$ being the position of the center of the $n$th barrier. We will focus on electronic states close to the bandgap and neglect nonparabolicity effects hereafter, so that a one-band Hamiltonian suffices to describe those states. Physical magnitudes of interest can be easily computed using a transfer-matrix formalism in this simple picture [12]. In particular, we can obtain subband energies under periodic boundary conditions and the Lyapunov coefficient, which measures the inverse of the localization length in units of the superlattice period. We will consider Thue-Morse superlattices (TMSLs) and Fibonacci superlattices (FSLs) since they have been already constructed starting from two basic building blocks $A$ and $A^{\prime}$ by means of

molecular beam epitaxy [1,2]. In our model, we take $A\left(A^{\prime}\right)$ consisting of a quantum-well of thickness $a\left(a^{\prime}\right)$ and a barrier of thickness $b$. ASLs are generated using the following inflation rules: $A \rightarrow A A^{\prime}, A^{\prime} \rightarrow A^{\prime} A$ for the TMSL and $A \rightarrow A A^{\prime}, A^{\prime} \rightarrow A$ for the FSL. In this way, finite and self-similar ASLs are obtained by $n$ successive applications of these rules, with $N=2^{n}$ wells in the TMSL case and $N=F_{n}$ wells in the FSL case. The Fibonacci numbers are generated from the recurrence law $F_{n}=F_{n-1}+F_{n-2}$, starting with $F_{0}=F_{1}=1$.

Interface roughness appears during growth in actual ASLs: Protrusions of one semicon- 
ductor into the other cause in-plane disorder and break translational invariance parallel to the layers. Because the in-plane average size of these defects depends on the growth conditions and it is unknown in most cases, one is forced to develop a simple approach. We carry out such an approach describing local excess or defect of monolayers by allowing $\Delta z_{n}$ to fluctuate uniformly around the nominal values $a+b$ or $a^{\prime}+b$. This approach enables us to restore the translational symmetry parallel to the layers, thus facilitating computations. Our approximation should be valid whenever the mean-free-path of electrons is much smaller than the in-plane average size of protrusions as electrons only see micro-quantum-wells with small area and uniform thickness. Therefore, each micro-quantum-well presents a slightly different value of its thickness and, as a consequence, resonant coupling between electronic states of neighbouring GaAs layers is decreased. To get an accurate description of electron dynamics, average over all possible configurations of disorder is indeed required because the number of interface defects as well as their mean thickness vary from layer to layer. For definiteness we take $\Delta z_{n}=a\left(1+W \epsilon_{n}\right)+b$ or $\Delta z_{n}=a^{\prime}\left(1+W \epsilon_{n}\right)+b$, where $W$ is a positive parameter measuring the maximum fluctuation and $\epsilon_{n}$ 's are distributed according to a uniform probability distribution $P\left(\epsilon_{n}\right)=1$ if $\left|\epsilon_{n}\right|<1 / 2$ and zero otherwise. Note that $\epsilon_{n}$ is a random uncorrelated variable, even when the lattice is constructed with the constraint that the mean values of $\Delta z_{n}$ follow the aperiodic sequences.

\section{RESULTS AND DISCUSSIONS}

We have studied GaAs-Ga $\mathrm{Ga}_{0.65} \mathrm{Al}_{0.35}$ As ASLs with $a=b=32 \AA$ and $a^{\prime}=35 \AA$. In this case the conduction-band offset is $250 \mathrm{meV}$ and the effective masses are $m_{\mathrm{GaAs}}^{*}=0.067 \mathrm{~m}$ and $m_{\mathrm{GaAlAs}}^{*}=0.096 m, m$ being the free-electron mass. In a periodic superlattice with $a^{\prime}=a=32 \AA$ only one allowed miniband lies below the barrier. We restrict ourselves to the study of the fragmentation of this miniband when aperiodicity is introduced. Averages over possible configurations of disorder comprised a number of realizations varying from 50 up to 100 to test the convergence of the computed mean values, and this convergence was always 
satisfactory. In order to compare with possible experimental situations, we have considered $W$ ranging from 0 up to a maximum of 0.08 . This value amounts to having maximum protrusion thicknesses of half a monolayer on average.

Figure [1(a) shows the dependence of the energy spectrum structure on the amount of disorder, $W$, present in a FSL with $N=F_{11}=144$ wells. The energy is measured from the conduction-band edge in GaAs. For a perfect $(W=0)$ FSL the overall structure of the energy spectrum is characterized by the presence of four main subbands. Inside each main subband the fragmentation pattern follows a trifurcation scheme in which each subband further splits from one to three subsubbands [13,14. Therefore, the energy spectrum of perfect and finite FSLs presents distinct pre-fractal signatures. The situation changes when randomness is introduced. In fact, although the tetrafurcation pattern of the perfect FSL still remain, the finer details corresponding to successive steps in the hierarchical splitting scheme are progressively smeared out on increasing the disorder due to growth fluctuations. Figure [ $1(\mathrm{~b})$ shows the dependence of the spectrum structure with the degree of disorder present in a TMSL with $N=128$ wells. For a perfect system the fragmentation scheme

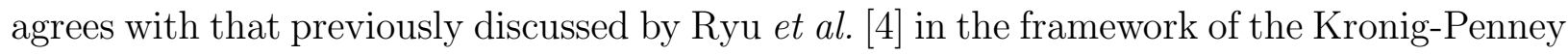
model, and displays pre-fractal signatures as well. The effects due to unintentional disorder are similar to those shown in Fig. I(a) for a FSL. In a previous paper we have demonstrated that pre-fractal signatures of FSLs can be properly described by considering the resonant coupling between electronic states of nearest-neighbor building blocks [14]. In this sense, the above results reinforce this conclusion for they show that the presence of the short-range disorder reduces the resonant coupling between quantum-wells and, consequently, weaken the physical mechanism giving rise to the self-similar pattern.

To investigate the effects due to the competition between long-range aperiodic order and short-range disorder on the transport properties, we have evaluated the localization length, $\ell$, as a function of the energy, a magnitude which can be readily determined numerically within the transfer matriz formalism [12,[15]. Figure 2(a) compares the localization length for a FSL with $N=F_{11}=144$ wells in two different cases. For the perfect $(W=0)$ 
case we obtain a very spiky structure. Each peak corresponds to a quasi-level, and most of them completely extends through the superlattice, its localization length being one order of magnitude greater than the system length. Note that the distribution of peaks reflects the overall fragmentation of the energy spectrum. On the contrary, when growth fluctuations are introduced, the localization length distribution becomes smoother and its value remains always smaller than the superlattice length, clearly revealing the onset of localization effects.

In Fig. 2(b) the same comparison is made for a TMSL with $N=128$ wells. Although the gross features of the plot, and their corresponding physical interpretation, are completely analogous to the case of the FSL, two interesting remarks are in order. In the first place, we observe that, in the perfect TMSL, there exists a significant number of states whose localization length is several orders of magnitude greater than the system size. This fact is related to the presence of latticelike states in the TMSL [1]. The occurrence of such states does not takes place in the FSL. In the second place, the mean value of $\ell$ is lower for the defective TMSL, hence indicating that the effects of localization are more intense in FSLs than they are for TMSLs with the same amount of disorder. A topic which has deserved some attention recently concerns the comparison between the transport properties in different kinds of aperiodically ordered structures. Our results indicate that, in presence of the same degree of growth fluctuations, TMSLs should exhibit better transport properties than FSLs. Finally, notice that the localization onset is more pronounced at the edges of the energy spectrum, meanwhile in the central regions of the spectrum (about $110 \mathrm{meV}$ ) the localization length almost equals the system size.

From Figs. 1 and 2 we can state that the general effect of disorder in ASLs is twofold. Firstly, it masks typical pre-fractal features smearing out the hierarchical splitting scheme taking place in perfect ASLs. This effect is independent of the precise nature of the underlying aperiodic order, this is to say, quasiperiodic (FSL) or self-similar but not quasiperiodic (TMSL). Secondly, the overall decrease of the localization length of the electronic states indicates that localization effects induced by random fluctuations are starting on.

To get a more complete understanding of the competition between long-range aperiodic 
order and random fluctuations, we have performed a bandwidth-scaling study [16]. To this end, we define the equivalent bandwidth $S$ as the sum of all allowed energy regions, thus being nothing but the Lebesgue measure of the energy spectrum, and compute it as a function of the number of wells $N$. The obtained results are presented in Fig. 3. Let us start discussing the scaling of the Fibonacci system. The equivalent bandwidth in the perfect system [Fig. 3(a)] decays as a power law of the form $S(N)=S_{0} N^{-\beta}$, where $S_{0} \sim 40 \mathrm{meV}$ is close to the bandwidth of the periodic superlattice and $\beta \sim 1 / 4$, within our numerical accuracy. According to earlier works [16], such a behaviour is characteristic of a fractal-like energy spectra, becoming a Cantor set in the limit $N \rightarrow \infty$. However, in the presence of moderate fluctuations ( $W \leq 0.05$ ), the equivalent bandwidth decays faster than a power law, as seen in Fig. 3(b) for $W=0.05$. The deviation from a power law is a natural consequence of the breaking of the self-similarity of the electronic spectrum 16n. Using a least square fit we have found that $S(N)=S_{0} N^{-\beta} \exp [-\alpha(W) N]$, where $\alpha(W) \sim 2 W^{2}$. From this expression two consequences can be drawn. First, the presence of the exponential factor indicates that, when disorder is introduced in an aperiodic system, its spectrum is not longer described in terms of a pure singular continuous component, whose presence is characterized by the presence of the power-law factor $N^{-\beta}$. Second, the competition between aperiodic longrange order and local disorder can be properly estimated from the deviation of $S$ from a pure power-law behaviour. Thus, localization effects dominate the electron dynamics in ASLs whose length is larger than the threshold length given by $N_{\text {th }}(W) \sim 1 / \alpha(W)$. This value is about $N_{\mathrm{th}} \simeq 200$ for $W=0.05$. Interestingly the deviation from power law decay becomes to be appreciable for moderately long systems, so that short imperfect ASLs will show energy spectra quite similar to that corresponding to a perfect ASL. This explains the success of previous experimental works to detect self-similar features in actual ASLs [11].

Let us now consider bandwidth scaling in TMSLs. As it was formerly discussed by Riklund et al. [17] the electronic properties of Thue-Morse lattices lie between those of quasiperiodic (Fibonacci) and usual periodic ones. This point has been further elaborated by Ryu et al. [4] who have shown that, attending to its scaling behaviour, the spectrum 
of TMSL can be decoupled into two components. One component scales as $N^{-\beta}$ with $\beta \rightarrow 0^{+}$, and corresponds to wave functions with a marked degree of extension (latticelike states). The other one also scales according to a power-law but $\beta>0$ in this case, and the corresponding charge density distribution of the related wave functions present large spatial fluctuations. According to this, the scaling behaviour shown in Fig. 3(a) can be easily understood. For short TMSLs the contribution of both kinds of critical states to the total bandwidth is essentially the same, for both spread uniformly over the whole sample in an analogous manner. But as the number of wells progressively increases, the contribution to $\mathrm{S}$ due to the second kind of wave functions decreases as a power law, whereas latticelike states give an almost constant contribution to $S$. As a consequence, only these states contribute significantly to the value of $S$ in the thermodynamic limit. The maximum number of wells considered in our computations has been $N=4096$, since the spectrum becomes too fragmented to compute accurately $S$ for larger systems. Conversely, the equivalent bandwidth $S$ decays exponentially when randomness takes place, as shown in Fig. 3(b). It is worth mentioning that asymptotic values of $S$ are almost the same for both kinds of disordered ASLs, indicating that the particular kind of long-range order of the underlying structure is immaterial with regard to their spatial extend.

\section{CONCLUSIONS}

In summary, we have proposed a realistic model to study actual aperiodic superlattices by allowing the quantum-well thicknesses to fluctuate around its nominal values in order to take into account interface roughness. Our results indicate that moderate fluctuations in the sequential deposition of layers have significant effects on both the energy spectrum and the spatial extension of wave functions. The fractal-like nature of an arbitrary spectrum is determined by two complementary features. In the first place, the energy spectrum becomes more and more fragmented as the ASL length grows. The physical origin for this fragmentation stems from resonant tunneling effects between electronic states of neighboring 
quantum-wells (short-range effects). In the second place, the splitting scheme of the energy spectrum must display a self-similar pattern. The physical origin for this self-similarity can be traced back to the structural self-similarity of the superlattice itself which, in turn, is imposed by the aperiodic ordering of the system (long-range effects). Taking both facts into account we conclude that the purported robustness of the aperiodic order present in ASLs does not suffices, by its own, to guarantee the fractal-like nature of the energy spectrum in the presence of disorder, because of the main effect of growth fluctuations is precisely to weaken the resonant coupling between electronic states. Hence, albeit the structural aperiodic order is preserved in the presence of moderate fluctuations, the self-similarity related to it can not be properly expressed, in its finer details, in the energy spectrum due to the loss of quantum coherence as a consequence of short-range effects.

The relative importance that this competition between long-range order and short-range disorder has on the transport properties depends critically on the length of the system. The values of the localization length we have obtained indicate that wave functions no longer spread over the whole ASL, as they do in the perfect case, but their degree of extension amounts a significant fraction of the system size in contrast with the usual view of localized states extending just over a few wells. Thus, as a final conclusion, we can state that distinctive features of a fractal-like spectrum can be experimentally observed in ALSs of practical interest, whose length is smaller than a threshold length. The value of this threshold length depends on the heterostructure quality attained during the growth process.

\section{ACKNOWLEDGMENTS}

Thanks are warmly due to Angel Sánchez for helpful discussions and critical reading of the manuscript. This work is supported by CICYT through project MAT95-0325. 


\section{REFERENCES}

[1] R. Merlin, K. Bajema, R. Clarke, F. -Y. Juang, and P. Bhattacharya, Phys. Rev. Lett. 55, 1768 (1985).

[2] R. Merlin, K. Bajema, J. Nagle, and K. Ploog, J. Phys. (Paris) Colloq. 48, C5-503 (1987).

[3] M. Kohmoto, L. P. Kadanoff, and C. Tang, Phys. Rev. Lett. 50, 1870 (1983).

[4] C. S. Ryu, G. Y. Oh, and M. H. Lee, Phys. Rev. B 48, 132 (1993).

[5] F. Domínguez-Adame, E. Maciá, B. Méndez, C. L. Roy and A. Khan, Semincond. Sci. Technol. 10, 797 (1995).

[6] A. Süto, J. Stat. Phys. 56, 525 (1989); J. Bellisard, B. Iochum, E. Scoppola, and D. Testard, Commun. Math. Phys. 125, 527 (1989).

[7] J. Bellisard, A. Bovier, and J. -M. Ghez, Commun. Math. Phys. 135, 379 (1991); Bovier and J. -M. Ghez, ibid. 158, 45 (1993).

[8] J. Todd, R. Merlin, R. Clarke, K. M. Mohanty, and J. D. Axe, Phys. Rev. Lett. 57, 1157 (1986).

[9] A. Chomette, B. Deveaud, A. Regreny, and G. Bastard, Phys. Rev. Lett. 57, 1464 (1986); L. Pavesi, E. Tuncel, B. Zimmermann, and F. K. Reinhart, Phys. Rev. B 39, 7788 (1989).

[10] Y. Liu and R. Riklund, Phys. Rev. B 35, 6034 (1987); S. Das Sarma and X. C. Xie, ibid. 37, 1097 (1989).

[11] D. Toet, M. Potemski, Y. Y. Wang, J. C. Maan, L. Tapfter, and K. Ploog, Phys. Rev. Lett. 66, 2128 (1991); S. Katsumoto, N. Sano, and S. Kobayashi, Solid State Commun. 85, 223 (1993); A. A. Yamaguchi, T. Saiki, T. Tada, T. Ninomiya, K. Misawa, and T. Kobayashi, ibid. 75, 955 (1990). 
[12] F. Domínguez-Adame, A. Sánchez, and E. Diez, Phys. Rev. B 50, 17736 (1994).

[13] E. Maciá, F. Domínguez-Adame, and A. Sánchez, Phys. Rev. B 49, 9503 (1994).

[14] E. Maciá, F. Domínguez-Adame, and A. Sánchez, Phys. Rev. E 50, 679 (1994).

[15] P. D. Kirkman and J. B. Pendry, J. Phys. C: Solid State Phys. 17, 4327 (1984).

[16] M. Kohmoto, Phys. Rev. Lett. 51, 1198 (1983); H. Hiramoto and M. Kohmoto, Phys. Rev. Lett. 62, 2714 (1989).

[17] R. Riklund, M. Severin, and Y. Liu, Int. J. Phys. B 1, 121 (1987). 


\section{FIGURES}

FIG. 1. Phase diagram for a (a) GaAs- $\mathrm{Ga}_{0.65} \mathrm{Al}_{0.35}$ As FSL with $N=F_{11}=144$ wells and (b) GaAs- $\mathrm{Ga}_{0.65} \mathrm{Al}_{0.35} \mathrm{As}$ TMSL with $N=128$ wells. Averages were taken over 10 realizations of the superlattice.

FIG. 2. Localization length for (a) a perfect $(W=0)$ GaAs- $\mathrm{Ga}_{0.65} \mathrm{Al}_{0.35} \mathrm{As}$ FSL with $N=F_{11}=144$ wells (solid line) as compared with a disordered FSL $(W=0.05)$ of the same length (dashed line), and (b) the same comparison for a GaAs-Ga $\mathrm{Ga}_{0.65} \mathrm{Al}_{0.35}$ As TMSL with $N=128$ wells. Averages were taken over 50 realizations of the superlattice.

FIG. 3. Equivalent bandwidth $S$ as a function of the number of wells in (a) perfect $W=0$ and (b) imperfect $W=0.05$ GaAs- $\mathrm{Ga}_{0.65} \mathrm{Al}_{0.35}$ As FSL (solid lines) and TMSL (dashed lines). Averages were taken over 50 realizations of the superlattice. 